\title{
2017 Acknowledgment of Reviewers
}

A peer-reviewed journal would not succeed without the dedication and insightful critiques of the reviewers whose efforts often go unrecognized. The editorial leadership at BioResearch Open Access is very grateful for the support of our expert peer reviewers who have donated their valuable time in 2017. We acknowledge and thank you for your service.

\author{
Ahmed Al Sabi \\ Sarah Blackstone \\ Ulrike Böer \\ Marco Cantini \\ John Cleland \\ Nick Conley \\ Alessandro Delitala \\ Carol DeSantis \\ Richard Deth \\ Aleksandar Jovanović \\ Kenji Kangawa \\ Amy Liu \\ Yasufumi Masaki
}

\author{
Mary Clare McCorry \\ Lina Mur \\ Patrice Poubelle \\ Decheng Ren \\ Yuki Saito \\ Kazumasu Sasaki \\ Annalucia Serafino \\ Yehuda Shoenfeld \\ T. G. Shrivastav \\ Daniel Stover \\ Marcelo Tolmasky \\ Kate Trajanoska \\ Hua-Tao Xie
}

